\title{
Study of Anxiety, Fear and Depression Associated with Breastfeeding in COVID-positive Mothers
}

\author{
Bhavya Doshi ${ }^{1} \odot$, Madhuri A Mehendale ${ }^{2}$, Arun H Nayak ${ }^{3}$, Archana A Bhosale ${ }^{4}$, Rashi Koria ${ }^{5}$
}

\begin{abstract}
Aim and objective: To study anxiety, fear and depression associated with breastfeeding in coronavirus disease (COVID)-positive mothers. Materials and methods: The following three standard scales were used to measure fear anxiety, and depression namely fear of coronavirus disease-2019 (COVID-19) scale (FCV-19S), corona disease anxiety scale (CDAS), and Edinburgh postnatal depression scale (EPDS) among postpartum women along with a self-made breastfeeding questionnaire by the author to assess the association with breastfeeding.

Results: Among the total of 62 respondents, a mean of 17 was obtained on the EPDS which is more than the cutoff value. FCV-19S and CDAS did not show extreme results except for few cases. The breastfeeding questionnaire suggested that most women are afraid of transmitting the infection to their newborns and they lack the knowledge about the importance of breast milk in warding off other infections. Also, women found it difficult to take care of their newborns on their own.

Conclusion: With this study, we could determine the effects of this pandemic on anxiety levels, depression, and fear of COVID infection in postpartum women. In the current scenario with the possibility of the next pandemic wave in India, there is an urgent need to provide emotional and psychosocial support to this group of the population during the crisis. Otherwise, the adverse outcome is possible involving both mother and newborn.

Clinical significance: The COVID-19 outbreak that began in China has turned into a pandemic that affects global health, thereby prompting the concentration of studies and clinical routines on treating and preventing the disease with measures like vaccination. Still, the clinical data regarding psychological manifestations of the ongoing pandemic among the general public, especially postpartum women and breastfeeding mothers, are inadequate. In this study, we analyzed experiences of breastfeeding mothers during the COVID-19 pandemic, specifically concerning how COVID-positive status affected their infant's feeding decisions. It clearly showed that being COVID positive created, affected, and exacerbated mental health issues for mothers.
\end{abstract}

Keywords: Anxiety, Breastfeeding, COVID-19, Depression, EPDS, Pandemic, Pregnancy.

Journal of South Asian Federation of Obstetrics and Gynaecology (2021): 10.5005/jp-journals-10006-1931

\section{INTRODUCTION}

An outbreak of a respiratory disease namely coronavirus disease-2019 (COVID-19) has rapidly disseminated worldwide, with a wide variety of clinical manifestations ranging from mild respiratory symptoms to severe pneumonia and a fatality rate ranging from $0.8 \%$ to even $9 \%$ in some countries. Interpersonal transmission is occurring both in the community and healthcare settings. ${ }^{2}$ The World Health Organization (WHO) classified the COVID-19 epidemic as a global pandemic on March 11, 2020. ${ }^{3}$

Coronavirus disease has presented the world to uncertainty and clinical dilemma with developing and constantly changing management guidelines and protocols. In the backdrop of this pandemic, it thus becomes crucial to study the effects of the infection on pregnancy, childbirth, and the postpartum period. Pregnant women do not appear to be at increased risk to contract the infection than the general population. ${ }^{4}$ Since pregnancy itself alters the body's physiology, including the immune system, and defense mechanism to counter infections in general, which can occasionally be attributed to more severe symptoms, this mechanism will be the same for COVID-19. ${ }^{5}$ Concerning vertical transmission (transmission from mother to baby antenatally or intrapartum), emerging evidence now suggests that vertical transmission is possible with low risk, although the proportion of pregnancies affected and the significance to the newborn is yet to be analyzed. At present, studies are suggesting recorded cases of vaginal secretions being tested positive for coronavirus. ${ }^{6}$ In infants,

\begin{abstract}
${ }^{1-5}$ Department of Obstetrics and Gynecology, Lokmanya Tilak Municipal Medical College and General Hospital, Mumbai, Maharashtra, India

Corresponding Author: Bhavya Doshi, Department of Obstetrics and Gynecology, Lokmanya Tilak Municipal Medical College and General Hospital, Mumbai, Maharashtra, India, Phone: +9109604912728, e-mail: doshibhavya.91@gmail.com

How to cite this article: Doshi B, Mehendale MA, Nayak AH, et al. Study of Anxiety, Fear and Depression Associated with Breastfeeding in COVID-positive Mothers. J South Asian Feder Obst Gynae 2021; 13(5):296-300.
\end{abstract}

Source of support: Nil

Conflict of interest: None

the risk of COVID-19 infection through breastfeeding is low, and the infection is usually mild or asymptomatic, while the consequences of not breastfeeding and separating the mother and the child can be significant. ${ }^{7}$

The postpartum period is often accompanied by maternal mental distress. Women are often concerned about their newborn's health.

Postnatal women with active COVID-19 infection should be managed with supportive care recommended for adults. ${ }^{8}$ Indian Council of Medical Research suggests that transmission after birth via contact with infectious respiratory secretions is a concern. ${ }^{9}$ Facilities are considering temporarily separating (e.g., separate rooms) the 
mother who has confirmed COVID-19 or pyrexia under investigation from her baby until the mother's transmission-based precautions are discontinued. The decision to discontinue the temporary separation of the mother from her baby should be made on a case-by-case basis after consultation with clinicians, public health officials, and infection prevention and control specialists. The decision should take into account disease severity, progression, signs and symptoms of the illness, and results of laboratory parameters for the virus that causes the COVID-19 infection in the mother and neonate. According to WHO, breastfeeding is particularly effective against infectious diseases because it strengthens the immune system by directly transferring antibodies from the mother. ${ }^{10}$ As with all confirmed or suspected COVID-19 cases, mothers with any signs and symptoms who are taking care of their baby, breastfeeding, or practicing skinto-skin contact should take precautions. As no relatives or caretakers are allowed in the COVID ward with patients, some pregnant and postpartum women may have fear, uncertainty, stress, or anxiety due to this disease.

So, we aim to study anxiety, fear and depression associated with breastfeeding in COVID-19 positive mothers.

\section{Materials and Methods}

\section{Study Description}

- Design: Prospective Cross-Sectional Observational

- Place of the study: Lokmanya Tilak Municipal Medical College and General Hospital, Sion, Mumbai, India

- Duration of the study: Period of 2 months

The duration of subject participation in this study was for a onetime questionnaire anytime between day 2 and day 42 during the postpartum period.

The study was conducted from July 1, 2020, to August 31, 2020.

- Sample size: A total of 62 COVID-positive postpartum mothers.

- Sampling method: Purposive sampling.

- Inclusión criteria: Subjects shall meet all the following inclusion criteria to be eligible for participation in this study.

All mothers delivering or registered with our center with the COVID-19 positive status in the postpartum period are breastfeeding.

Exclusion criteria: (i) Mothers without a COVID-19 infection. (ii) Mothers who are not breastfeeding due to any other reasons.

- Standard scales for measurement: The anxiety, fear and depression were assessed using the standard corona disease anxiety scale (CDAS), fear of COVID-19 scale (FCV-19S) and Edinburgh postpartum depression scale (EDPS), respectively during the hospital stay in the postpartum period. Breastfeeding experience and knowledge were assessed by a predesigned, pretested questionnaire designed by the author.

\section{Scales}

\section{The Edinburgh Postpartum Depression Scale}

The EPDS was created by Cox et al. ${ }^{11}$ and was translated into Hindi manually for the purpose of this study and accordingly validated. The scale is rated on a four-point Likert scale and consists of 10 items. The scale ranging from 0 to 3 is intended to evaluate postpartum depression levels experienced by women. The lowest total score that can be obtained is 0 , with the highest score being 30 . Individuals with a score of 13 or more are considered at higher risk of developing depression.

\section{Fear of COVID-19 Scale}

FCV-19S, measuring the emotional fear reactions toward the COVID-19 pandemic, consists of seven items and its scoring is on a five-point Likert-type scale from 1 to 5 . A higher level of fear is determined by a sum of these scores which is from 7 to 35 . Designing and testing the validity and reliability of this tool were done by Ahorsu et al. in Iran in 2020. ${ }^{12}$

\section{Corona Disease Anxiety Scale}

CDAS is an 18-item tool in which each participant receives a score from 0 to 54. This tool measures the corona-related anxiety in two aspects, namely psychological symptoms and physical symptoms, and the items are answered on a Likert scale from 0 to 3 . The validity and reliability of this questionnaire have been assessed in Alipour et al. ${ }^{13}$

\section{Questionnaire for Breastfeeding COVID-positive Mothers}

It is a 24-question-based tool to test the basic knowledge of the mother about COVID-19, the precautions to be taken while breastfeeding, and any problems that the mother faces while breastfeeding and taking care of her newborn on her own. This is a pretested, predesigned questionnaire created by the author.

The approval is taken from the ethical committee for the same.

\section{Result}

The total number of deliveries at our institute during the study period was 912 and COVID-19 positive deliveries were 62, making the incidence $6.8 \%$ over the study period of 2 months.

\section{Demographic Characteristics}

Demographic characteristics of postpartum women are mentioned in Table 1.

The mean age of the study population was 26.11 years. Among the participants, the maximum had completed graduation that is $29.03 \%$. More participants were multigravida that is $58.06 \%(n=36$, study group). Among the participants, more were evaluated on day 2 of delivery which was $37.09 \%(n=23)$. The number of participants post a cesarean section delivery was more $(53.22 \%)$ than post a normal vaginal delivery.

Table 1: Demographic characteristics

\begin{tabular}{|c|c|c|c|}
\hline Variable & $(M e a n+/-S D)$ & Total & Percentage \\
\hline Age (year) & $26.11 \pm 3.44$ & - & - \\
\hline \multicolumn{4}{|l|}{ Education } \\
\hline - Primary & & 6 & 9.67 \\
\hline · High school & & 8 & 12.90 \\
\hline - Higher secondary (12th) & & 16 & 25.80 \\
\hline - Graduate & & 18 & 29.03 \\
\hline - Postgraduate & & 14 & 22.58 \\
\hline \multicolumn{4}{|l|}{ Gravida } \\
\hline - Primigravida & & 26 & 41.93 \\
\hline - Multigravida & & 36 & 58.06 \\
\hline \multicolumn{4}{|l|}{ Day of delivery } \\
\hline - Day 1 & & 20 & 32.26 \\
\hline - Day 2 & & 23 & 37.09 \\
\hline - $\geq$ Day 3 & & 19 & 30.64 \\
\hline \multicolumn{4}{|l|}{ Mode of delivery } \\
\hline - Vaginal delivery & & 29 & 46.77 \\
\hline - Cesarean section & & 33 & 53.22 \\
\hline
\end{tabular}




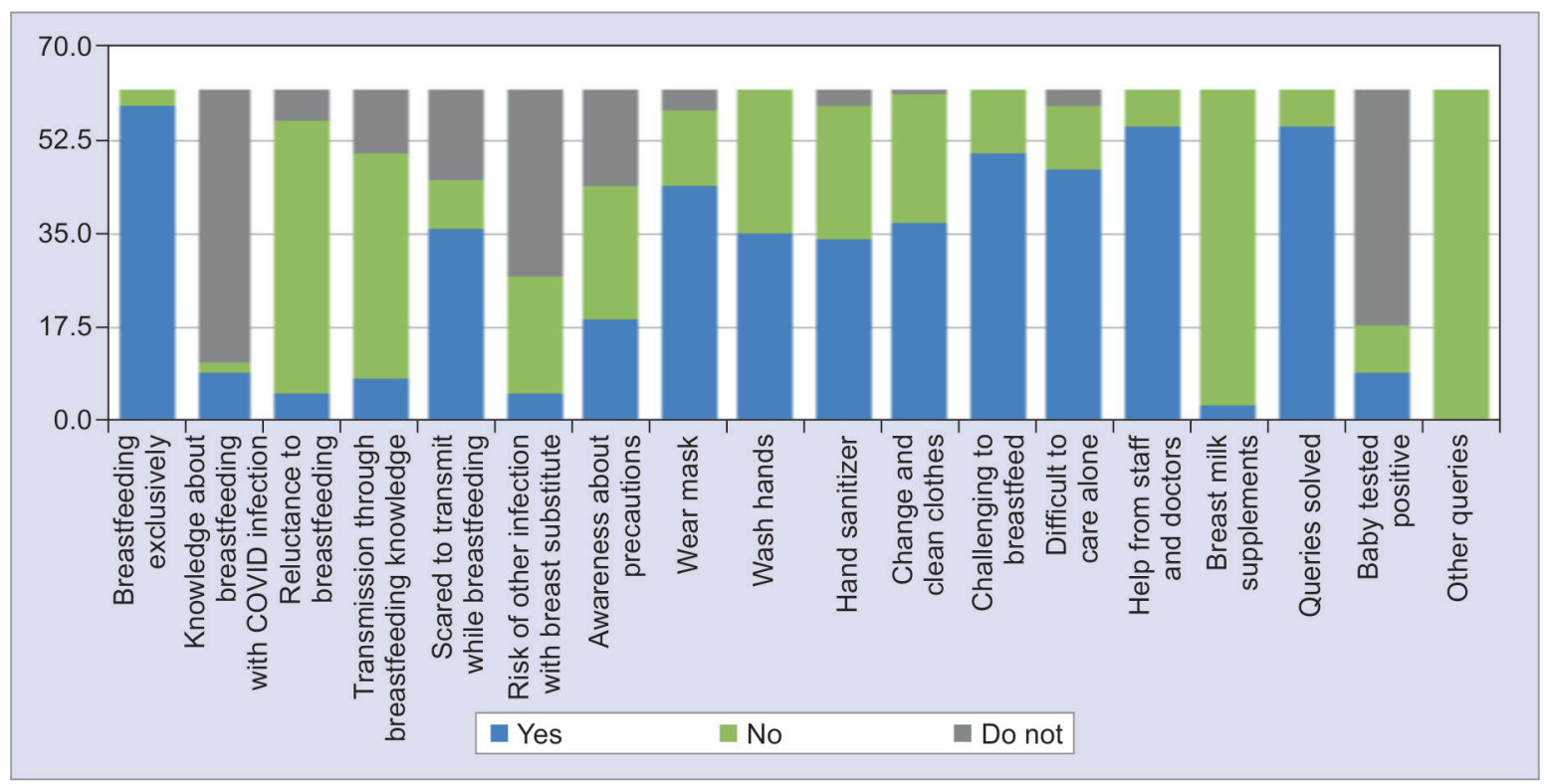

Fig. 1: Breastfeeding questionnaire data

Table 2: Mean and SD of EDPS, FCV-19S, and CDAS

\begin{tabular}{llc}
\hline & Mean & Std deviation \\
\hline EDPS & 17.53 & 3.05 \\
FCV-19S & 17.5 & 5.27 \\
CDAS & 15.93 & 5.16 \\
\hline
\end{tabular}

\section{Mean and Standard Deviation}

Mean and standard deviations of EDPS, FCV-19S, and CDAS are depicted in Table 2.

Among the participants, 91.9\% ( $n=57$, case group) scored higher than 13 on EDPS.

The mean score of EDPS was $17.53 \pm 3.05$ which is more than the standard cutoff value of 13 for the same. The mean score of FCV-19S and CDAS was $17.5 \pm 5.27$ and $15.93 \pm 5.16$, respectively.

\section{Breastfeeding Questionnaire Data}

Figure 1 depicts the response of case participants with respect to breastfeeding and issues regarding the same.

As evident from the figure given below, 59 mothers were breastfeeding their babies exclusively. Among the participants, maximum $(n=51)$ were doubtful about spreading a COVID infection through breastfeeding but most of them ( $n=51$, study group) were not reluctant to breastfeed with their COVID-positive status. Awareness about the rarity of transmission of COVID infection through breast milk was reported by only $12.9 \%$ of the participants $(n=8)$. Fear of transmitting infection through breast milk was reported by $58 \%$ of the participants $(n=36)$. The risk of COVID vs other infections due to a breast milk substitute was a worry for $8 \%$ of participants while $56.4 \%$ were not aware of such a risk. Totally $30 \%$ of the participants were aware of precautions; 44 participants were using a mask, 35 were following handwashing, and 34 were also using a hand sanitizer before handling the newborn. Totally $59.67 \%$ of the participants reported changing and cleaning their clothes regularly. Among the participants, $80.64 \%$ of women found it challenging to breastfeed their baby in the immediate postoperative period and $75.8 \%$ found it difficult to breastfeed and take care of their newborn alone. Totally $88.7 \%$ of the participants said "yes" to receiving help from staff and doctors in breastfeeding their baby. Only three participants said "yes" to giving supplements to their newborn in the form of infant formula, with the reason being mostly advice from the doctor or staff. Totally 55 women said that their queries regarding breastfeeding were resolved. Most women did not know about the COVID status of their newborns. Women confirmed that all other queries regarding the same were resolved.

\section{Discussion}

The COVID-19 pandemic is one of the most dangerous threats of the 21st century, and every government is waging war against it. The entire world's attention is on the present global outbreak, which has ramifications in nearly every country. As a result of this crisis, scientists began investigating the therapy and prevention of this disease right away. Psychological effects on vulnerable populations, such as pregnant women, are unavoidable when such a traumatic life event occurs. The ongoing pandemic has stoked severe worry and panic, underscoring the growing importance of mental care. ${ }^{14}$ The problem is that there is a scarcity of information on the psychological effects of outbreaks. Only a few studies have looked into the psychological consequences of COVID-19 on the general public ${ }^{15}$ and healthcare personnel, ${ }^{16}$ and current research and clinical practices are mostly focused on treating and preventing it due to its high mortality and morbidity rates.

After the outbreak of this epidemic, it is imperative that we have a serious discussion about certain pressing concerns. An example of a crucial issue is postpartum care and lactation. For the same reason, Capanna et al. prepared a useful guide that can be used by all obstetricians and gynecologists to help them deal with the crucial difficulties for pregnant women who are COVID-19 positive. The research done in Italy was based on both theoretical and empirical evidence. ${ }^{17}$ Iran posed the first real hazard to pregnant women's mental health when it comes to 
Study of Anxiety, Fear and Depression Associated with Breastfeeding in COVID-positive Mothers

dealing with their stress during pregnancy. During the COVID-19 epidemic, Mirzadeh and Khedmat spoke about the various aspects of pregnancy and the postpartum period. This has shown that pregnant and postpartum women need psychological help throughout this period. ${ }^{18}$

It has been found that FCV-19S and CDAS correlate closely in an Iranian study by Salehi et al. ${ }^{19}$ However, pregnant women were shown to have higher rates of depressive episodes on the EPDS and higher levels of anxiety on the CDAS. Depression is a well-documented and prevalent consequence of pregnancy and the postpartum period. An estimate from the WHO found that roughly $10 \%$ of pregnant women suffer from a mental illness, primarily depression. In developing countries, the prevalence of this illness is higher, reaching $15.6 \%$ during pregnancy and $19.8 \%$ after childbirth (WHO). ${ }^{20}$ Perinatal depression (EDPS $>13$ ) was found in $43.3 \%$ of patients in a study by Kale et al., and in $91 \%$ of patients in our study. ${ }^{21}$ Using standardized scales, we found that during pandemics, the prevalence of mental disorders was significantly higher than in normal circumstances.

To begin, many women are misinformed about the safety of COVID-19 and breastfeeding, with newborns being taken from their mothers or mothers not permitted to nurse when they experience COVID symptoms after birth, which is a huge worry. The pandemic's early stages saw several countries routinely separating mothers and babies, with breast milk substitutes occasionally being recommended. ${ }^{22}$ Similarly, the American Academy of Pediatrics recommended separating the mother and child if a case was suspected ${ }^{23}$ - a recommendation that has since been modified (AAP, 2020). Despite the fact that several organizations in the UK have said that breastfeeding is safe and should be encouraged (UNICEF UK, 2020), healthcare professionals and worried parents may have nonetheless been misled by these erroneous statements. This was made worse by the transmission of false information on social media, which was widespread and had a variety of effects on health management during the epidemic. ${ }^{24}$ If a lockdown occurs again, policymakers and our health system must provide prompt, accurate, and visible support for promoting breastfeeding among healthcare providers and on social media platforms. Keeping a mother and child apart is not only against nature but also goes against all we know about encouraging the commencement of breastfeeding. ${ }^{25}$ Stable neonatal behavior is facilitated by letting the mom and baby stay in the same room. ${ }^{26}$ Despite the fact that our study found no evidence of women being misinformed about the safety of breastfeeding or advised against sharing a room with their infant, the fear of spreading COVID led some women to stop breastfeeding, while others experienced depression and anxiety over their baby's health.

We do know, however, that vertical transmission during pregnancy or after birth is extremely rare, with newborns having very low infection rates and a low chance of progressing to more serious diseases and problems. ${ }^{27}$ Breast milk, vaginal fluids, and cord blood were tested negative for the virus in a study by Kapadia et al. Even if the woman delivered vaginally, no nasopharyngeal swabs from the baby were positive at birth, supporting no vertical transmission of COVID-19 during the third trimester of the pregnancy. ${ }^{28}$ According to a case study, an asymptomatic woman who took preventive steps, such as wearing a mask, using a hand sanitizer, and washing her hands, did not infect her newborn. ${ }^{29}$ $\mathrm{WHO}$ also recommends these safety precautions for asymptomatic mothers. Mothers who took precautions when touching their babies accounted for over $75 \%$ of those in our survey.

The consequences of not nursing are frequently neglected in talks about breastfeeding safety during the pandemic. Breastfeeding benefits both the newborn and the mother in the long run. ${ }^{30}$ If a mother contracts an infection during breastfeeding, her body's immune system protects the newborn through her breast milk. ${ }^{31}$ After being exposed to COVID-19, if a mother does not show any symptoms and subsequently exposes her kid to it, it is more likely that breast milk will protect the newborn. Besides this, breastfeeding benefits the health of the mother both physiologically (by lowering inflammation and improving sleep) and psychologically (by reducing stress). ${ }^{32}$

\section{CONCLUSION}

During the COVID-19 epidemic, this study examined women's breastfeeding and newborn care experiences, specifically how their COVID-positive status influenced their nursing and rooming-in decisions. Postpartum moms who were tested positive for COVID saw an increase in their mental health concerns, including anxiety and mood disorders. Overall, these findings are relevant for policymakers who are striving to support breastfeeding and should be utilized to reflect on the provision of maternal mental support in future instances of this type. When comparing the benefits and hazards of breastfeeding vs not breastfeeding, the big picture must be considered. A woman's mental health problems are exacerbated even further while caring for a newborn on her own. A mother's mental health is preserved when she meets her own nursing objectives. But when she does not-particularly if she runs into problems or fears infection-her risk of anxiety, melancholy, and grief rises.

\section{Clinical Significance}

A conundrum develops as to how we might move forward in changing things for the better. Rooming-in and face-to-face nursing assistance are not going to return to the pre-pandemic levels any time soon, with some families needing to shield for longer. Also, moms who live in cramped quarters suffer from loneliness and are forced to care for their newborns alone because of rooming-in. It is likely that for the foreseeable future, counseling, coaching, and follow-up services will rely heavily on online and telephone support. It is critical to catch any indicators of mental illness as soon as possible. We must also ensure that all mothers receive accurate and positive information and support services in order to break down the barriers some are facing.

\section{ORCID}

Bhavya Doshi 우 https://orcid.org/0000-0001-8423-7846

\section{References}

1. John Hopkins University and Medicine, coronavirus.jhu.edu [Internet], Coronavirus Resource Center. 2021. Available from: https:// coronavirus.jhu.edu/data/mortality.

2. Mackenzie JS, Smith DW. COVID-19: a novel zoonotic disease caused by a coronavirus from China: what we know and what we don't. Microbiol Aust 2020:MA20013. DOI: 10.1071/MA20013.

3. Cucinotta D, Vanelli M. WHO declares COVID-19 a pandemic. Acta Biomed 2020;91(1):157-160. DOI: 10.23750/abm.v91i1.9397.

4. Available from: https://www.rcog.org.uk/globalassets/documents/ guidelines/2020-07-24-coronavirus-covid-19-infection-in-pregnancy.pdf. 
5. Zhao X, Jiang Y, Zhao Y, et al. Analysis of the susceptibility to COVID-19 in pregnancy and recommendations on potential drug screening. Eur J Clin Microbiol Infect Dis 2020:1-12. DOI: 10.1007/s10096-020-03897-6.

6. Schwartz A, Yogev Y, Zilberman A, et al. Detection of severe acute respiratory syndrome coronavirus 2 (SARS-CoV-2) in vaginal swabs of women with acute SARS-CoV-2 infection: a prospective study. BJOG 2021;128(1):97-100. DOI: 10.1111/1471-0528.16556.

7. World Health Organization. Breastfeeding and COVID-19: scientific brief (23 June 2020). Geneva, Switzerland: World Health Organization; 2020.

8. Chawla D, Chirla DI, Dalwai S, et al. Perinatal-neonatal management of COVID-19 infection-guidelines of the Federation of Obstetric and Gynecological Societies of India (FOGSI), National Neonatology Forum of India (NNF), and Indian Academy of Pediatrics (IAP). Indian Pediatr 2020;57(6):536-548. DOI: 10.1007/s13312-020-1852-4.

9. Poon LC, Yang H, Kapur A, et al. Global interim guidance on coronavirus disease 2019 (COVID-19) during pregnancy and puerperium from FIGO and allied partners: information for healthcare professionals. Int J Gynecol Obstet 2020;149(3):273-286. DOI: 10.1002/ ijgo.13156.

10. Hanson LA. Breastfeeding provides passive and likely long-lasting active immunity. Ann Allergy Asthma Immunol 1998;81(6):523-537. DOI: 10.1016/S1081-1206(10)62704-4.

11. Cox JL, Holden JM, Sagovsky R. Detection of postnatal depression: development of the 10-item Edinburgh Postnatal Depression Scale. Br J Psychiatry 1987;150(6):782-786. DOI: 10.1192/bjp.150.6.782.

12. Ahorsu DK, Lin CY, Imani V, et al. The fear of COVID-19 scale: development and initial validation. Int J Mental Health Addict 2020:1-9. DOI: 10.1007/s11469-020-00270-8.

13. Alipour A, Ghadami A, Alipour Z, et al. Preliminary validation of the Corona disease anxiety scale (CDAS) in the Iranian sample. Health Psychol J 2020;8(32):163-175. DOI: 10.30473/HPJ.2020.52023.4756.

14. Xiang YT, Yang Y, Li W, et al. Timely mental health care for the 2019 novel coronavirus outbreak is urgently needed. Lancet Psychiatry 2020;7(3): 228-229. DOI: 10.1016/S2215-0366(20)30046-8.

15. Wang C, Pan R, Wan X, et al. Immediate psychological responses and associated factors during the initial stage of the 2019 coronavirus disease (COVID-19) epidemic among the general population in China. IJERPH 2020;17(5):1729. DOI: 10.3390/ijerph17051729.

16. Li Z, Ge J, Yang M, et al. Vicarious traumatization in the general public, members, and non-members of medical teams aiding in COVID-19 control. Brain Behav Immun 2020;88:916-919. DOI: 10.1016/j.bbi.2020.03.007. PII: S0889-1591(20)30309-3.

17. Capanna F, Haydar A, McCarey C, et al. Preparing an obstetric unit in the heart of the epidemic strike of COVID-19: quick reorganization tips. J Matern Neonatal Med 2020:1-7. DOI: 10.1080/ 14767058.2020 .1749258$.

18. Mirzadeh M, Khedmat L. Pregnant women in the exposure to COVID-19 infection outbreak: the unseen risk factors and preventive healthcare patterns. J Matern Neonatal Med 2020:1-2. DOI: 10.1080/14767058.2020.1749257.

19. Salehi L, Rahimzadeh M, Molaei E, et al. The relationship among fear and anxiety of COVID-19, pregnancy experience, and mental health disorder in pregnant women: a structural equation model. Brain Behav 2020;10(11):e01835. DOI: 10.1002/brb3.1835.

20. World Health Organisation. www.who.int [internet], maternalmental-health. 2021. Available from: https://www.who.int/teams/ mental-health-and-substance-use/maternal-mental-health.

21. Kale DP, Nadkarni TK, Pawar AP, et al. A prospective observational study of the effects of COVID-19 pandemic on mental health status of COVID-positive pregnant women in a COVID Care Center. J South Asian Fede Obst Gynae 2021. DOI: 10.5005/jp-journals-100061904.

22. Tomori C, Gribble K, Palmquist $A E$, et al. When separation is not the answer: breastfeeding mothers and infants affected by COVID-19. Maternal Child Nutr 2020;16(4):e13033. DOI: 10.1111/mcn.13033.

23. Bartick MC, Schwarz EB, Green BD, et al. Suboptimal breastfeeding in the United States: maternal and pediatric health outcomes and costs. Matern Child Nutr 2017;13(1):e12366. DOI: 10.1111/ men.12366.

24. Singh $P$, Singh $S$, Sohal $M$, et al. Psychological fear and anxiety caused by COVID-19: insights from Twitter analytics. Asian J Psychiatr 2020;54:102280. DOI: 10.1016/j.ajp.2020.102280.

25. Pérez-Escamilla R, Hall Moran V. Scaling up breastfeeding programmes in a complex adaptive world. Matern Child Nutr 2016;12(3):375-380. DOI: 10.1111/mcn.12335.

26. Ahn SY, Ko SY, Kim KA, et al. The effect of rooming-in care on the emotional stability of newborn infants. Korean J Pediatr 2008;51(12):1315-1319. DOI: 10.3345/kjp.2008.51.12.1315.

27. Lokken EM, Walker CL, Delaney S, et al. Clinical characteristics of 46 pregnant women with a severe acute respiratory syndrome coronavirus 2 infection in Washington State. Am J Obstet Gynecol 2020;223(6):911.e1. DOI: 10.1016/j.ajog.2020.05.031.

28. Kapadia SN, Mehta A, Mehta CR, et al. Study of pregnancy with COVID-19 and its clinical outcomes in a tertiary care teaching hospital in Western India. J South Asian Feder Obs Gynae 2021;13(2):125-130. DOI: 10.5005/jp-journals-10006-1886.

29. Lowe B, Bopp B. COVID-19 vaginal delivery-a case report. Aust N Z J Obstet Gynaecol 2020;60(3):465-466. DOI: 10.1111/ajo.13173.

30. Victora CG, Bahl R, Barros AJ, et al. Breastfeeding in the 21st century: epidemiology, mechanisms, and lifelong effect. Lancet 2016;387(10017):475-490. DOI: 10.1016/S0140-6736(15)01024-7.

31. Garofalo RP, Goldman AS. Expression offunctional immunomodulatory and anti-inflammatory factors in human milk. Clin Perinatol 1999;26(2):361-377. DOI: 10.1016/s0095-5108(18)30057-5.

32. Brown A, Shenker N. Experiences of breastfeeding during COVID-19: lessons for future practical and emotional support. Matern Child Nutr 2021;17(1):e13088. DOI: 10.1111/mcn.13088. 